\title{
NOUVELLe
}

\section{Déficits motivationnels et acides gras polyinsaturés}

Fabien Ducrocq ${ }^{1,2}$, Pierre Trifilieff $^{1}$
${ }^{1}$ Université de Bordeaux, INRAE, Bordeaux INP Aquitaine, NutriNeuro,

146 rue Léo Saignat, 33000 Bordeaux, France.

${ }^{2}$ Department of Translational Neuroscience, Brain Center Rudolf Magnus, University Medical Center Utrecht, Universiteitsweg 100, 3584 Utrecht, Pays-Bas. F.Y.C.Ducrocq@umcutrecht.nl

\section{Maladies psychiatriques et déficits motivationnels}

De nombreuses maladies psychiatriques, telles que la schizophrénie, les troubles bipolaires ou la dépression majeure ont une origine développementale et multifactorielle, à la fois génétique et environnementale. Bien qu'elles soient considérées comme différentes d'un point de vue clinique [1], elles partagent certains symptômes dont les substrats neurobiologiques pourraient être similaires [2]. Plusieurs études indiquent d'ailleurs l'existence de facteurs communs, génétiques (polymorphismes génétiques) [3] ou environnementaux [4], qui pourraient y contribuer. Une telle approche, fondée sur l'identification de biomarqueurs communs, est désormais privilégiée par la communauté scientifique, comme en témoigne la création, en 2010, du programme Research domain criteria initiative (RDoC) des National Institutes of Health (NIH) aux États-Unis [5]. Ce cadre a pour objectif d'influencer les méthodes de recherche sur les maladies psychiatriques en se fondant, non plus sur l'observation clinique d'un ensemble de symptômes (les catégories diagnostiques du Diagnostic and statistical manual of mental disorders, DSM), mais sur l'identification des mécanismes physiopathologiques qui en sont à l'origine, dans une démarche «translationnelle» $[5]$.

Nous sommes partis du constat que les maladies psychiatriques précédemment citées comportent toutes un dysfonctionnement du système de récompense et de motivation, un des domaines principaux définis par le programme
$\mathrm{RDoC}^{\mathrm{l}}$, en lien avec une altération de la transmission dopaminergique [2]. Ces perturbations sont en relation directe avec l'apparition de symptômes, tels que l'avolition², l'apathie ou l'anhédonie ${ }^{3}$, qui gênent considérablement les patients dans leur vie quotidienne. Dans l'étude que nous avons conduite, nous avons tenté de déterminer si l'«amotivation » présente dans différentes maladies pourrait résulter de mécanismes pathogéniques communs.

\section{Maladies psychiatriques et déficience} en acides gras polyinsaturés (AGPI)

Nous nous sommes intéressés aux modifications du métabolisme lipidique décrites chez certains patients atteints de schizophrénie, de troubles bipolaires ou de dépression majeure, en particulier à la diminution des taux d'acides gras polyinsaturés (AGPI) de type n-3 [6]. $\varepsilon n$ effet, bien que certaines études cliniques décrivent des effets bénéfiques de la supplémentation en AGPI n-3 sur certains symptômes de ces maladies [7-10], ces résultats sont controversés, et l'implication du statut lipidique dans le développement de ces symptômes reste très peu étudiée. Les $A G P I$ sont des constituants essentiels des membranes cellulaires, dont ils modulent les propriétés physico-chimiques (Figure 1). Ils interagissent avec certaines protéines membranaires, et peuvent également agir comme des messagers

${ }^{1}$ www.nimh.nih.gov/research/research-funded-by-nimh/rdoc/ definitions-of-the-rdoc-domains-and-constructs.shtml

${ }^{2}$ Incapacité à initier et persister dans des activités dirigées vers un but (selon le DSM, $5^{e}$ édition).

${ }^{3}$ Incapacité d'un sujet à ressentir des émotions positives lors de situations de vie pourtant considérées antérieurement comme plaisantes. secondaires. Dans le cerveau, où ils sont particulièrement abondants, ils s'accumulent principalement pendant le développement périnatal et jouent un rôle structurel très précoce. Les mammifères sont incapables de synthétiser de novo certains AGPI, notamment les $A G P I n-3$, qui sont donc qualifiés d'《essentiels », car leur présence dans l'organisme dépend exclusivement de l'apport alimentaire.

Le but de l'étude publiée dans la revue Cell Metabolism [11] était d'établir s'il pourrait exister un lien causal entre la déficience en AGPI n-3 observée dans des maladies psychiatriques, telles que la schizophrénie, les troubles bipolaires, ou la dépression majeure, et certains phénotypes neurobiologiques et comportementaux qui les caractérisent. Notre hypothèse était que la déficience en AGPI n-3 pourrait induire un dysfonctionnement de la transmission dopaminergique mésolimbique ${ }^{4}$ conduisant, du point de vue comportemental, à un déficit de motivation.

Déficience en AGPI n-3 et troubles de la motivation: dysfonctionnement du système mésolimbique

Dans cette étude, la déficience en AGPI n-3 a été induite chez la souris par manipulation nutritionnelle. À partir de l'accouplement, les femelles ont été soumises à un régime alimentaire contrôle ou déficient en AGPI n-3. Au sevrage, la descendance est maintenue sous ces mêmes régimes, jusqu'à l'âge adulte. Nous avons évalué

\footnotetext{
${ }^{4}$ La voie mésolimbique transmet la dopamine de l'aire tegmentale ventrale (ATV) située au niveau du mésencéphale, vers le striatum ventral.
} 


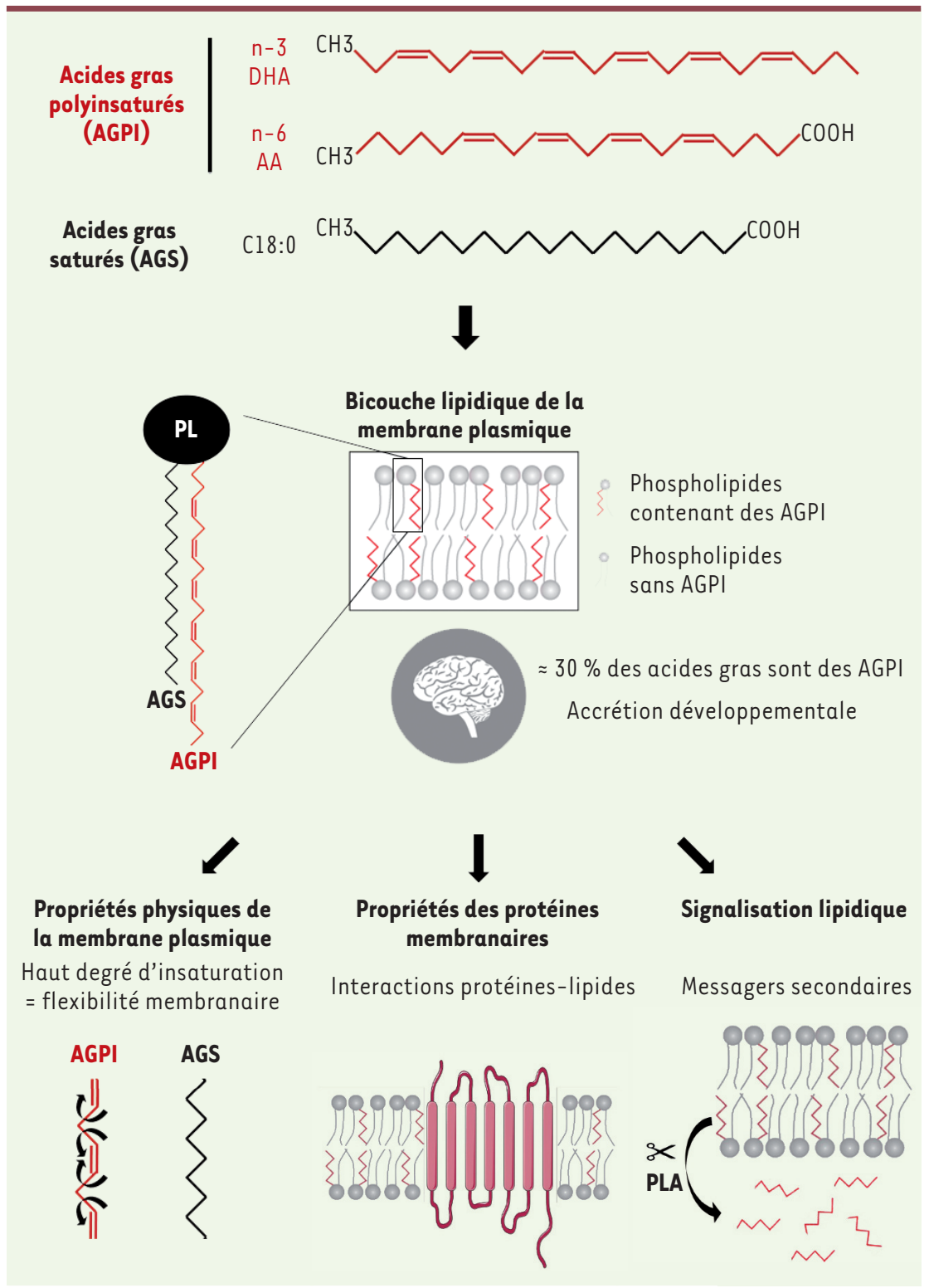

Figure 1. Les acides gras polyinsaturés et leurs fonctions cellulaires. $\varepsilon$ n haut : représentation des structures moléculaires des principaux acides gras polyinsaturés (AGPI) de la famille $\mathrm{n}-3$ (acide docosahexaénoïque, DHA) et $\mathrm{n}-6$ (acide arachidonique, $A A$ ), présentant respectivement six et quatre double-liaisons («insaturations ») sur leur chaîne carbonée, en comparaison à un acide gras saturé (AGS : ici acide stéarique, $\mathrm{Cl} 8: 0$ ) qui n'en contient aucune (dans la nomenclature $n-x$ des AGPI, $x$ indique la position de la première double liaison sur la chaîne carbonée, à partir de l'extrémité méthyle). Au milieu: représentation d'un phospholipide ( $\mathrm{PL}$ ) membranaire contenant deux acides gras, I'un saturé (en noir) et l'autre polyinsaturé (en rouge). La membrane plasmique contenant ces phospholipides comporte un degré d'«insaturation », qui dépend de la quantité des AGPI qui la composent et de la famille ( $n-3$ ou n-6, entre autres) à laquelle ils appartiennent. Ces AGPI (principalement le DHA et l'AA) sont particulièrement concentrés dans les cellules du système nerveux central. Ils sont dit « essentiels » du fait de l'impossibilité pour notre organisme de les synthétiser de novo. Leur origine est donc exclusivement alimentaire, et leur accumulation a lieu en grande partie au cours du développement périnatal. En bas : les AGPI jouent principalement trois rôles dans la membrane plasmique: 1) Leur degré d'insaturation influe sur la flexibilité membranaire. En effet, la longueur de la chaîne et le nombre des doubles liaisons déterminent la hauteur, la forme et la flexibilité d'un acide gras. Les AGPI sont donc hautement flexibles, pouvant adopter différentes conformations. Ils vont ainsi contribuer à la courbure, la fluidité et l'épaisseur de la bicouche lipidique de la membrane plasmique. Les flèches de part et d'autre des doubles liaisons de l'AGPI représentent ces liens flexibles. 2) L'environnement lipidique membranaire affecte l'activité des protéines transmembranaires. En effet, les AGPI favorisent le changement de conformation de certains récepteurs, voire agissent comme des «ligands » sur des sites spécifiques (e.g. la rhodopsine, qui comporte une « poche de liaison » dans laquelle se fixe le DHA, ce qui facilite alors la phototransduction). 3) Les AGPI incorporés dans les phospholipides membranaires peuvent être hydrolysés par les phospholipases A (PLA) et agir comme messagers secondaires sur différentes voies de signalisation.

l'intégrité des processus motivationnels dans des tâches de conditionnement opérant $^{5}$ permettant d'évaluer la motivation (i.e., la capacité de l'animal à effectuer un

${ }^{5}$ Le conditionnement opérant est un concept selon lequel un comportement est renforcé (c'est-à-dire que sa reproduction est rendue plus probable) en raison de la conséquence de ce comportement. Dans le cas présent, l'utilisation d'une récompense alimentaire permet d'étudier les comportements produits dans le but de l'obtenir, et d'en isoler les différentes composantes telles que la motivation. effort, c'est-à-dire à appuyer sur un levier pour obtenir une récompense alimentaire palatable, face à une demande motivationnelle croissante). Les paradigmes de mesure de la motivation face à l'effort sont un outil puissant pour modéliser et étudier les dysfonctionnements motivationnels caractéristiques de multiples maladies neurologiques et psychiatriques. En effet, la notion d'effort pour obtenir une récom- pense a été définie par le programme RDoC comme un facteur majeur dans l'étude du système « à valence positive $»^{6}$. Nous avons pu mettre en évidence, chez la sou-

\footnotetext{
6 Système responsable des réponses de l'organisme à des situations ou à des contextes "positifs", tels que la recherche et la consommation de récompenses, l'apprentissage de comportements dirigés vers un but, etc. (https://www.nimh.nih.gov/research/research-funded-bynimh/rdoc/constructs/positive-valence-systems.shtml).
} 


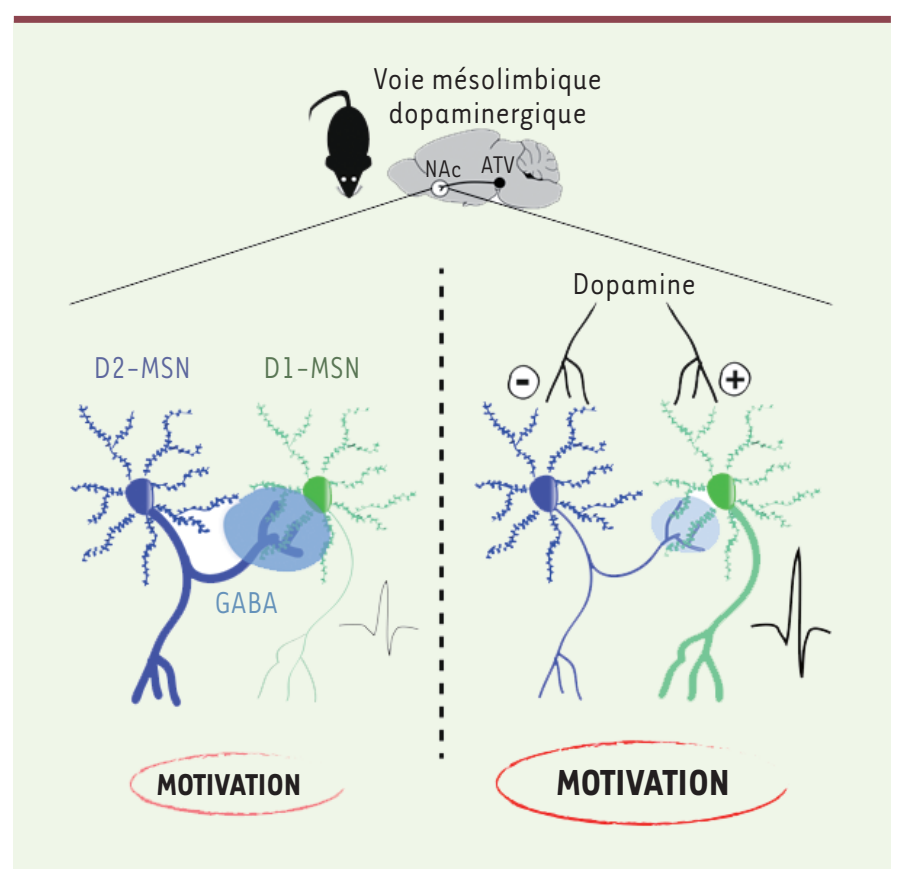

Figure 2. Représentation schématique de la transmission mésolimbique dopaminergique dans le noyau accumbens du cerveau chez la souris. Ce schéma montre la connectivité entre les neurones épineux moyens (medium spiny neurons, MSN) exprimant les récepteurs D1 (D1-MSN) et D2 (D2-MSN) de la dopamine dans le noyau accumbens (NAc), structure sous contrôle dopaminergique, ainsi que leur implication dans les processus motivationnels. Les MSN sont des neurones inhibiteurs GABAergiques. Les D2-MSN inhibent différentes cibles incluant, localement, des DI-MSN (inhibition latérale). La dopamine (DA) libérée par des neurones de l'aire tegmentale ventrale (ATV) module l'activité des MSN : d'une part, elle inhibe les D2-MSN, ce qui entraîne notamment une levée de l'inhibition latérale, et, d'autre part, elle active les Dl-MSN. Du point de vue comportemental, il en résulte une facilitation des processus motivationnels. GABA : acide $\gamma$-aminobutyrique.

ris adulte, la présence d'un déficit de la motivation induit par une déficience en AGPI n-3 durant le développement périnatal, qui peut être partiellement prévenu par une supplémentation en AGPI n-3 à la naissance, mais pas au sevrage.

Par des techniques d'électrophysiologie ex vivo, nous avons montré que ce déficit motivationnel s'accompagnait d'un dysfonctionnement des neurones épineux moyens (medium spiny neurons, MSN) du noyau accumbens, une population neuronale qui joue un rôle essentiel dans la modulation des processus motivationnels (Figure 2). En effet, nous avons découvert que l'excitabilité des neurones DI-MSN exprimant le récepteur dopaminergique de type $\mathrm{Dl}$ (RDl) était diminuée, conséquence d'une augmentation de la transmission GABAergique «latérale » inhibitrice issue des neurones D2-MSN exprimant le récepteur dopaminergique de type D2 (RD2) (Figure 3).

Afin de démontrer l'implication des AGPI dans ces déficits neurobiologiques et comportementaux, nous avons utilisé un modèle de souris transgénique caractérisé par l'expression conditionnelle (dépendant de la recombinase $\mathrm{Cre}$ ) de l'acide gras désaturase FATl du nématode Caenorhabditis elegans dans certaines populations neuronales. Cette enzyme permet la conversion des AGPI n-6 (présents en plus grande quantité du fait de la carence en AGPI n-3) afin de restaurer des taux d'AGPI n-3 appropriés uniquement dans les populations neuronales ciblées par la manipulation génétique. Nous avons montré que l'expression de FATl, durant la période périnatale, dans les cellules cérébrales exprimant RD2 - mais pas dans celles exprimant RDI était suffisante pour normaliser le déficit de motivation et restaurer les propriétés électrophysiologiques des neurones MSN du noyau accumbens dans une situation de déficience en AGPI n-3 (Figure 3).

\section{Perspectives}

Ces résultats constituent la première démonstration de l'existence d'un lien causal entre des modifications du taux d'AGPI dans une sous-population neuronale spécifique et une altération comportementale. Nous avons montré que les neurones D2-MSN du noyau accumbens présentent une sensibilité particulière à la manipulation des taux d'AGPI. II reste à découvrir quelles sont les bases cellulaires et moléculaires de cette vulnérabilité. Par ailleurs, les résultats de notre étude suggèrent que la diminution des taux d'AGPI décrite dans plusieurs maladies psychiatriques contribue directement à l'expression de symptômes d' «amotivation», dont elle pourrait constituer un biomarqueur prédictif.

Les causes de la déficience en AGPI n-3 observée chez les patients atteints des maladies psychiatriques concernées restent à identifier. Cependant, cette déficience pourrait être liée à l'existence de polymorphismes dans la séquence nucléotidique des gènes codant les enzymes du métabolisme des phospholipides et des AGPI (e.g., protéines de liaison aux acides gras, acide gras désaturases, phospholipases), plutôt qu'au seul déséquilibre alimentaire en acides gras [12]. Une prédisposition génétique pourrait donc être responsable, au moins en partie, de la diminution des taux d'AGPI n-3 observée chez les patients, mais les liens entre ces polymorphismes génétiques et le statut en AGPI des patients atteints de ces maladies psychiatriques restent à établir. $\diamond$

Motivational deficits and polyunsaturated fatty acids

\section{LIENS D'INTÉR̂̂T}

Les auteurs déclarent n'avoir aucun lien d'intérêt concernant les données publiées dans cet article. 


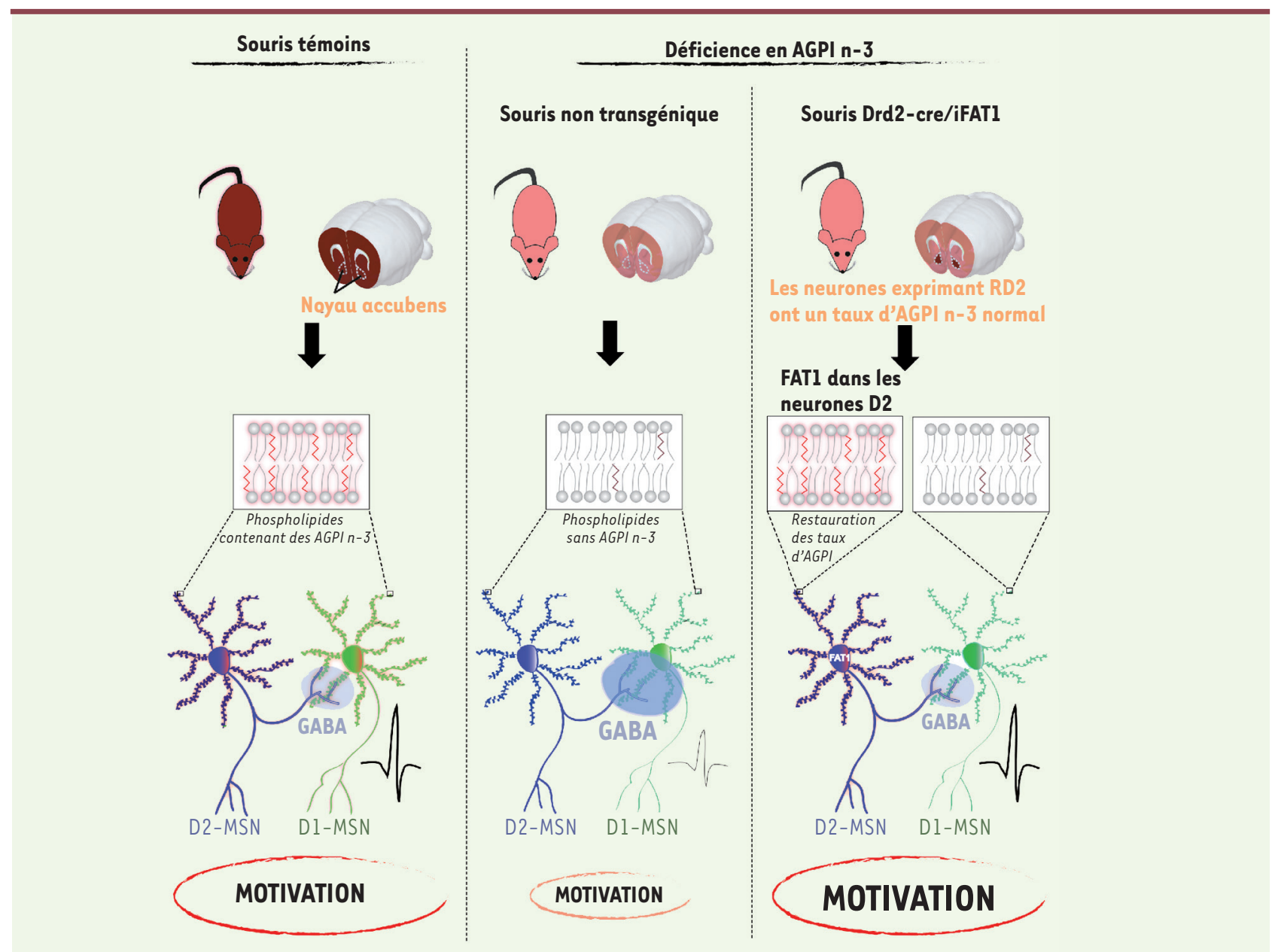

Figure 3. Synthèse des principaux résultats présentés dans l'article [11]. La déficience développementale en acides gras polyinsaturés (AGPI) du type n-3 chez la souris perturbe la transmission mésolimbique dans le noyau accumbens (NAc), notamment en raison d'une connectivité anormale entre neurones dopaminoceptifs (MSN), cette perturbation s'accompagnant d'un déficit motivationnel. L'utilisation d'un modèle de souris transgénique, Drd2-Cre/iFATl, caractérisé par une expression sélective de l'acide gras désaturase FATl dans les neurones possédant le récepteur D2 de la dopamine (RD2), a permis de montrer que le maintien des taux d'AGPI n-3 dans ces neurones, alors que les autres types cellulaires restaient déficients pour ces AGPI, était suffisant pour prévenir l'apparition des altérations neurobiologiques ainsi que le déficit motivationnel induit par la déficience développementale en AGPI n-3. D1-MSN : neurone épineux moyen exprimant le récepteur D1 de la dopamine ; D2-MSN : neurone épineux moyen exprimant le récepteur D2 de la dopamine ; GABA : acide $\gamma$-aminobutyrique.

\section{RÉFÉRENCES}

1. Diagnostic and statistical manual of mental disorders : DSM-5. Fifth edition. Arlington, VA : American Psychiatric Publishing, 2013.

2. Whitton AE, Treadway MT, Pizzagalli DA. Reward processing dysfunction in major depression, bipola disorder and schizophrenia. Curr Opin Psychiatry $2015 ; 28: 7-12$.

3. Gandal MJ, Haney JR, Parikshak NN, et al. Shared molecular neuropathology across major psychiatric disorders parallels polygenic overlap. Science 2018 ; $359: 693-7$.

4. Nestler $\varepsilon$ J, Peña CJ, Kundakovic M, et al. Epigenetic basis of mental illness. The Neuroscientist 2016 ; 22 : 447-63.
5. Insel T, Cuthbert B, Garvey M, et al. Research domain criteria (RDoC): toward a new classification framework for research on mental disorders. Am J Psychiatry $2010 ; 167: 748-51$.

6. Messamore $\varepsilon$, McNamara RK. Detection and treatment of omega-3 fatty acid deficiency in psychiatric practice: rationale and implementation. Lipids Health Dis $2016 ; 15: 25$.

7. Amminger GP, Schäfer MR, Schlögelhofer M, et al. Longer-term outcome in the prevention of psychotic disorders by the Vienna omega-3 study. Nat Commun $2015 ; 6: 7934$.

8. Amminger GP, Nelson B, Markulev C, et al. The Neurapro biomarker analysis: long-chain omega-3 fatty acids improve 6-month and 12-month outcomes in youths at ultra-high risk for psychosis. Biol Psychiatry $2020 ; 87: 243-52$.
9. Sarris J, Mischoulon D, Schweitzer I. Omega-3 for bipolar disorder: meta-analyses of use in mania and bipolar depression. J Clin Psychiatry 2012 ; 73 : 81-6.

10. Grosso G, Pajak A, Marventano S, et al. Role of omega-3 fatty acids in the treatment of depressive disorders: a comprehensive meta-analysis of randomized clinical trials. PLoS One 2014 ; 9 : e96905.

11. Ducrocq F, Walle R, Contini A, et al. Causal link between $n-3$ polyunsaturated fatty acid deficiency and motivation deficits. Cell Metab $2020 ; 31$ : 755-72.e7.

12. Hamazaki K, Maekawa M, Toyota T, et al. Fatty acid composition of the postmortem prefrontal cortex of patients with schizophrenia, bipolar disorder, and major depressive disorder. Psychiatry Res 2015 ; 227 : 353-9. 